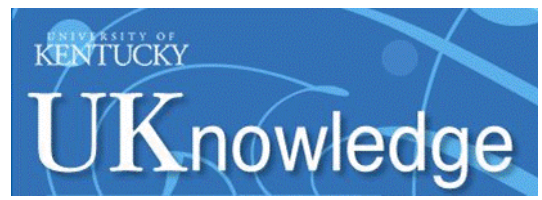

University of Kentucky

UKnowledge

\title{
Comparing Adult Cannabis Treatment-Seekers Enrolled in a Clinical Trial with National Samples of Cannabis Users in the United States
}

\author{
Erin A. McClure \\ Medical University of South Carolina \\ Jacqueline S. King \\ The Emmes Corporation \\ Aimee Wahle \\ The Emmes Corporation \\ Abigail G. Matthews \\ The Emmes Corporation \\ Susan C. Sonne

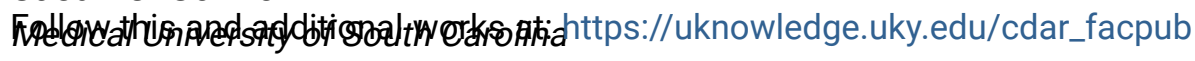 \\ Part of the Behavior and Behavior Mechanisms Commons, and the Substance Abuse and Addiction \\ seemext page for additional authors \\ Right click to open a feedback form in a new tab to let us know how this document benefits you.
}

\section{Repository Citation}

McClure, Erin A.; King, Jacqueline S.; Wahle, Aimee; Matthews, Abigail G.; Sonne, Susan C.; Lofwall, Michelle R.; McRae-Clark, Aimee L.; Ghitza, Udi E.; Martinez, Melissa; Cloud, Kasie; Virk, Harvir S.; and Gray, Kevin M., "Comparing Adult Cannabis Treatment-Seekers Enrolled in a Clinical Trial with National Samples of Cannabis Users in the United States" (2017). Center on Drug and Alcohol Research Faculty Publications. 4.

https://uknowledge.uky.edu/cdar_facpub/4

This Article is brought to you for free and open access by the Drug and Alcohol Research at UKnowledge. It has been accepted for inclusion in Center on Drug and Alcohol Research Faculty Publications by an authorized administrator of UKnowledge. For more information, please contact UKnowledge@lsv.uky.edu. 


\section{Comparing Adult Cannabis Treatment-Seekers Enrolled in a Clinical Trial with National Samples of Cannabis Users in the United States}

Digital Object Identifier (DOI)

https://doi.org/10.1016/j.drugalcdep.2017.02.024

Notes/Citation Information

Published in Drug and Alcohol Dependence, v. 176, p. 14-20.

(C) 2017 Elsevier B.V. All rights reserved.

This manuscript version is made available under the CC-BY-NC-ND 4.0 license

https://creativecommons.org/licenses/by-nc-nd/4.0/.

The document available for download is the author's post-peer-review final draft of the article.

\section{Authors}

Erin A. McClure, Jacqueline S. King, Aimee Wahle, Abigail G. Matthews, Susan C. Sonne, Michelle R. Lofwall, Aimee L. McRae-Clark, Udi E. Ghitza, Melissa Martinez, Kasie Cloud, Harvir S. Virk, and Kevin M. Gray 


\title{
Comparing adult cannabis treatment-seekers enrolled in a clinical trial with national samples of cannabis users in the United States
}

\author{
Erin A. McClure ${ }^{1, *}$, Jacqueline S. King ${ }^{2}$, Aimee Wahle ${ }^{2}$, Abigail G. Matthews $^{2}$, Susan C. \\ Sonne ${ }^{1}$, Michelle R. Lofwall ${ }^{3}$, Aimee L. McRae-Clark ${ }^{1}$, Udi E. Ghitza ${ }^{4}$, Melissa Martinez ${ }^{5}$, \\ Kasie Cloud $^{6}$, Harvir S. Virk, and Kevin M. Gray ${ }^{1}$ \\ ${ }^{1}$ Department of Psychiatry and Behavioral Sciences, Medical University of South Carolina, \\ Charleston SC \\ ${ }^{2}$ The Emmes Corporation, Rockville MD \\ ${ }^{3}$ Center on Drug and Alcohol Research, University of Kentucky College of Medicine, Lexington KY \\ ${ }^{4}$ National Institute on Drug Abuse Center for the Clinical Trials Network, Rockville MD \\ ${ }^{5}$ Department of Psychiatry, University of Texas Health Science Center at San Antonio, San \\ Antonio TX
}

${ }^{6}$ CODA, Inc., Portland OR

\section{Abstract}

Background-Cannabis use rates are increasing among adults in the United States (US) while the perception of harm is declining. This may result in an increased prevalence of cannabis use disorder and the need for more clinical trials to evaluate efficacious treatment strategies. Clinical trials are the gold standard for evaluating treatment, yet study samples are rarely representative of the target population. This finding has not yet been established for cannabis treatment trials. This study compared demographic and cannabis use characteristics of a cannabis cessation clinical trial sample (run through National Drug Abuse Treatment Clinical Trials Network) with three nationally representative datasets from the US; 1) National Survey on Drug Use and Health, 2) National Epidemiologic Survey on Alcohol and Related Conditions-III, and 3) Treatment Episodes Data Set - Admissions.

\begin{abstract}
Methods-Comparisons were made between the clinical trial sample and appropriate cannabis using sub-samples from the national datasets, and propensity scores were calculated to determine the degree of similarity between samples.

Results-Results showed that the clinical trial sample was significantly different from all three national datasets, with the clinical trial sample having greater representation among older adults, African Americans, Hispanic/Latinos, adults with more education, non-tobacco users, and daily and almost daily cannabis users.
\end{abstract}

* Corresponding Author Erin A. McClure, Ph.D., Assistant Professor, Medical University of South Carolina, 125 Doughty St., Suite 190, MSC 861, Charleston, SC 29425, Phone: 843-792-7192, mccluree@ musc.edu. 
Conclusions-These results are consistent with previous studies of other substance use disorder populations and extend sample representation issues to a cannabis use disorder population. This illustrates the need to ensure representative samples within cannabis treatment clinical trials to improve the generalizability of promising findings.

\section{Keywords}

Cannabis; marijuana; cannabis use disorder; treatment; clinical trial; sample representativeness; generalizability

\section{Introduction}

Cannabis is the most commonly used illicit substance in the United States (US) (Center for Behavioral Health Statistics and Quality, 2015). Cannabis use rates are increasing among adults (Grucza et al., 2016; Hasin et al., 2016), while the perception of harm associated with cannabis is declining (Berg et al., 2015; Johnston et al., 2015; Pacek et al., 2015; Sinclair et al., 2013). Legislation surrounding cannabis use and possession is rapidly changing in the US, and while the full impact of this change is still largely unknown, it may contribute to an increase in chronic use and cannabis-related harms (Hall and Lynskey, 2016). While the public perception of cannabis is changing, the literature on the adverse health and societal effects of cannabis is growing (Brady and Li, 2014; Compton et al., 2014; Hall, 2009; Hall and Degenhardt, 2009; Lynskey and Hall, 2000; Meier et al., 2012; Volkow et al., 2016). The development of cannabis use disorder (CUD) is a potential adverse effect that may occur among chronic users. Data comparing rates of cannabis use and CUD prevalence from 2001-2002 to 2012-2013 showed a more than doubling of cannabis use rates and prevalence of CUD among adults, with three out of 10 users developing a CUD (Hasin et al., 2015). This study found that the risk of developing a CUD did not increase, but CUD rates increased due to the increased prevalence of use in the US.

Given the apparent increased prevalence of cannabis use among adults, healthcare settings and substance use disorder (SUD) treatment centers may encounter more adults seeking treatment for CUD or obtaining court-mandated treatment. While research focused on the treatment of CUD is needed, the generalizability of results from clinical trials does not always translate well to real-world practice settings (Humphreys, 2016; Rothwell, 2005). Clinical trials often employ stringent exclusion criteria and tend to recruit small and unrepresentative sub-samples of the target population. A recent analysis explored the representativeness of study samples within the National Drug Abuse Treatment Clinical Trials Network (CTN) compared to national US datasets (Susukida et al., 2016). The 10 CTN studies used in this analysis varied with respect to the intervention being tested and particular substance of abuse being targeted by the intervention, though none were focused specifically on cannabis use. This study found notable differences in the demographics of those participating in research studies versus those entering SUD treatment, specifically in the areas of education and employment. It is unknown, however, if similar differences exist among those with CUD when compared to nationally representative samples. One study found that the majority of adults meeting criteria for cannabis dependence through a household survey would have been excluded from a clinical trial using common exclusion 
criteria (Okuda et al., 2010). Therefore, the representativeness of CUD clinical trial samples is a timely issue given that novel therapeutics and treatment strategies are needed for CUD and more treatment trials are likely in the near future. It is important to identify differences, should they exist, among those who volunteer for randomized clinical trials compared to other cannabis users in order to improve sample representativeness and the generalizability of promising clinical trial results.

In order to address the question of sample representativeness in CUD clinical trials, this study aimed to compare the demographics and cannabis use characteristics of adult participants with CUD enrolled in a multi-site treatment trial for cannabis cessation with a comparable population from national datasets. This clinical trial was conducted within the CTN and recruited a geographically diverse sample through six study sites in the US. The study sample was compared with two unique national samples of adults; 1) meeting criteria for CUD (or cannabis dependence) through household surveys, and 2) entering publically funded SUD treatment programs for CUD.

\section{Methods}

\subsection{Participants and Study Procedures}

This multi-site clinical trial evaluated a pharmacotherapy added to a behavioral intervention for cannabis cessation among US adults (Achieving Cannabis Cessation: Evaluating $\mathrm{N}$ Acetylcysteine Treatment [ACCENT]). Methodological details for this study can be found elsewhere (McClure et al., 2014). Briefly, participants were adult men and women ( $\mathrm{N}=302)$ between the ages of 18-50 years who met criteria for cannabis dependence (based on Diagnostic and Statistical Manual of Mental Disorders (DSM IV (First, 1994)), were interested in quitting, and had a positive urine cannabinoid test during the screening assessment. Exclusion criteria were focused on safety concerns and aimed to appropriately characterize the sample. The following exclusion criteria were employed: known allergy to $\mathrm{N}$-Acetylcysteine (NAC), pregnant or lactating, use of NAC-containing supplements or hazardous concurrent medications, current enrollment in treatment for cannabis dependence, use of synthetic cannabinoids, current substance dependence other than nicotine or caffeine and/or positive urine drug screen (other than cannabis), on opioid-replacement therapy, recent history of asthma, uncontrolled medical or psychiatric illness that could put the participant at risk, and risk of homicide or suicide.

Eligible participants were randomized to receive NAC or matched placebo for 12 weeks. Contingency management procedures were used for both experimental groups to reinforce abstinence from cannabis during twice weekly study visits, in addition to a separate compensation schedule targeting attendance at study visits. Six study sites across the US participated in the ACCENT trial (Behavioral Health Services of Pickens County [Pickens, SC], The APT Foundation [New Haven, CT], University of Kentucky Medical Center [Lexington, KY], University of California, Los Angeles Integrated Substance Abuse Programs [Los Angeles, CA], The University of Texas Health Science Center at San Antonio [San Antonio, TX], and CODA, Inc. [Portland, OR]). This trial was registered with Clinicaltrials.gov (NCT01675661), and completed study procedures in August 2015. The institutional review boards at participating centers approved the study protocol, which was 
overseen by an independent National Institute on Drug Abuse-appointed Data and Safety Monitoring Board. Cannabis abstinence outcomes from this trial are described elsewhere (Gray et al., Under Review).

\subsection{Measures}

Sources of Data—Data from the ACCENT study were compared to three nationally representative datasets. Due to some constraints introduced by the national datasets, six ACCENT participants were excluded from this analysis. Four participants were 50 years of age (national datasets include 50 as the lower limit of a larger range). Two participants had less than an eighth grade education, leading to an insufficient comparator group. This resulted in a final sample of 296 participants being included from the ACCENT study in the current analysis.

Three national datasets were compared with the ACCENT study sample: the National Survey on Drug Use and Health (NSDUH), the National Institute on Alcohol Abuse and Alcoholism (NIAAA) 2012-2013 National Epidemiologic Survey on Alcohol and Related Conditions-III (NESARC-III), and the Treatment Episodes Data Set - Admissions (TEDSA). The NSDUH is a publicly available dataset that measures the prevalence of drug use in the US among a representative community dwelling population. NSDUH 2014 data were used for this analysis (United States Department of Health and Human Services Substance Abuse and Mental Health Services Administration, 2016). Weighting variables were used for this dataset as recommended for the NSDUH. The NESARC-III includes data from noninstitutionalized, civilian adults (18 years or older) in the US. This survey employed multistage probability sampling to choose respondents. A limited dataset from the NESARC-III was used for the current analysis. Additional details regarding the NESARCIII are available elsewhere (Grant et al., 2014). Weighting variables were used for this dataset. The TEDS-A includes data on treatment admissions (including court-mandated admissions) to SUD programs that are publicly funded. We used the most recent TEDS-A data available at the time, which was from 2013 (Substance Abuse and Mental Health Services Administration; Center for Behavioral Health Statistics and Quality, 2015). Since the ACCENT study only enrolled those who were cannabis dependent, had used cannabis in the past 30 days, and were between the ages of 18-50; appropriate sub-samples were selected from the national samples based on those variables (i.e., meeting criteria for cannabis dependence or CUD [based on DSM-IV or DSM-5 criteria] of any severity level and past 30 day use of cannabis [estimated based on past year use for the NESARC-III dataset] and within the age of inclusion). The sample sizes from each data source are shown in Table 1 for weighted and unweighted samples of the total records available and the cannabis use sub-samples.

Demographics—Basic demographic information was obtained for all datasets. Response options were collapsed for consistency across datasets, mostly for the ACCENT study sample. The following demographic variables were used in the current analysis with the following response categories: age (NSDUH: 18-20, 21-23, 24-29, 30-34, 35-49; NESARC-III and TEDS-A: 18-20, 21-24, 25-29, 30-34, 35-39, 40-44, 45-49), sex (male, female), race (Caucasian, African American, Other), ethnicity (Hispanic or Latino, non- 
Hispanic or Latino), education (9-11 years, 12 years or equivalent [no diploma, high school graduate, GED or equivalent], >12 years), employment (working now, unemployed, or other [not in the labor force, disability]), and marital status (never married, married/domestic partnership, separated/divorced/widowed).

Cannabis and Tobacco Use-Measures of cannabis use and history included: age of first use (11 and under, 12-14, 15-17, 18-20, 21 and over) and days of use in the past month (NSDUH: continuous; NESARC-III and TEDS-A: 1-3 times in the past month, 1-2 times in the past week, 3-6 times in the past week, or daily use). Tobacco use was also compared. Tobacco use data was not available for the TEDS-A dataset. Tobacco use was categorized based on use in the past month (daily tobacco user, non-daily tobacco user, no use in the past month).

\subsection{Statistical Analyses}

Categorical variables from the national samples were compared to ACCENT variables using a Pearson chi-squared test, accounting for the sample weight for comparisons of ACCENT to NSDUH and NESARC-III. Frequencies were calculated using the sample weight for NSDUH and NESARC-III. A Wilcoxon rank sum test was conducted to compare the continuous variable, number of days of cannabis use in the prior 30 days, for ACCENT and NSDUH, adjusting for sample weight.

Among the four datasets, there was minimal missing data (see Table 2 for rates of missing or unknown data for each variable) and thus individuals with missing data were excluded from the propensity score analyses. All available data was utilized for the chi-squared tests reported in Table 2. The ACCENT study had three participants with missing data for race (1\%). The NSDUH and NESARC-III national datasets had missing data imputed and information was obtained from the respective codebooks for each survey (Grant et al., 2014; United States Department of Health and Human Services Substance Abuse and Mental Health Services Administration, 2016). The NESARC-III dataset only have missing values for the derived variables of past month cannabis use (0.8\%) and current tobacco use (6.2\%). Missing values were not imputed in the TEDS-A dataset, and therefore had the most missing data ranging from $0.4 \%$ for age of first THC use to $7 \%$ for marital status.

Propensity scores were calculated by modeling the probability of a participant being enrolled in ACCENT, based upon characteristics collected at baseline of ACCENT and the national sample. These characteristics included age, gender, race, ethnicity, education, employment, marital status, and age at first cannabis use. Propensity scores were calculated individually for each national dataset compared to ACCENT. For NSDUH, propensity scores were also computed based on number of days of cannabis use in the past month and cigarette smoking status. The baseline characteristics considered for NESARC-III also included cannabis use in the past month and cigarette smoking status. Propensity scores for TEDS-A were additionally based upon cannabis use in the past month. Logistic regression was used to calculate the propensity scores for TEDS-A, and logistic regression adjusted for the sample weight for NSDUH and NESARC-III. 
Introduced by Stuart and colleagues (Stuart et al., 2001), the propensity score-based index, denoted $\Delta \mathrm{p}$, assesses the similarity of clinical trial samples compared to target populations. This propensity score-based index $\Delta \mathrm{p}$ was computed as the difference between the average propensity scores of ACCENT and the national sample. $\Delta \mathrm{p}$ was standardized by dividing by the pooled standard deviation of the propensity scores and can be used as an index of similarity between samples with regards to all baseline characteristics used to compute the propensity score. A difference of more than 0.25 standard deviations (standardized $\Delta \mathrm{p}$ ) in propensity score mean values indicates significant differences between the clinical trial and the target population (Cochran and Rubin, 1973; Rosenbaum and Rubin, 1985; Stuart, 2010).

Two-sample $t$-tests were also conducted for comparison of the propensity scores between ACCENT and the national sample, adjusted by sample weight for NSDUH and NESARCIII, and unadjusted for TEDS-A. All analyses were performed with SAS software, Version 9.4 of the SAS System for Windows (SAS Institute Inc., Cary, NC, USA), using the FREQ procedure to calculate the chi-squared test and Wilcoxon rank sum test, and the LOGISTIC procedure for logistic regression. The TTEST procedure was used for the $t$-test for the comparison of ACCENT and TEDS-A, and the PROBT function was used to calculate the $p$ value for the weighted comparisons of ACCENT to NSDUH and NESARC-III.

\section{Results}

\subsection{Sample Comparisons}

Demographics-All comparisons to the ACCENT study sample are shown in Table 2. Generally, the participants in the ACCENT trial were older, with less representation among younger users (ages 18-20) and more representation among the older age group (45-49 for NESARC-III and TEDS-A; 35-49 for NSDUH). All datasets showed greater numbers of men being represented compared to women (nearly two-thirds were men), with differences in gender distribution between ACCENT and NESARC-III data (fewer men and more women in NESARC-III). Racial comparisons showed that among the household surveys (NSDUH and NESARC-III), ACCENT had more representation of African Americans, but lower proportions of other racial categories. TEDS-A data appeared to be more evenly mixed among Caucasian and African Americans, but less representative of other races and differed from ACCENT for all racial comparisons. ACCENT had a greater proportion of Hispanic and Latino participants compared to all national samples, and participants in ACCENT were also significantly more likely to have $>12$ years of education than the three national datasets. Compared to household survey data, ACCENT participants had lower rates of employment; though employment rates were higher for ACCENT when compared to the TEDS-A data. Finally, the ACCENT sample had higher rates of married participants (or domestic partnership) and lower rates of never married participants compared to NSDUH and TEDS-A (but not NESARC-III).

Cannabis and tobacco use-Age of first cannabis use was generally comparable across samples, with the exception of ACCENT having slightly higher rates of first use beyond the age of 21 compared to the NSDUH dataset. Participants in the ACCENT sample had a 
significantly higher mean number of days of cannabis use in the 30 days prior compared to NSDUH data (26.0 days versus 20.9 days). They were also significantly less likely to have used cannabis 1 to 3 times in the past month and 1 to 2 times per week in the last month than those in NESARC-III and TEDS-A. The ACCENT sample had higher rates of daily or almost daily cannabis use. Finally, lower rates of daily tobacco use were found in ACCENT compared to national samples (NSDUH and NESARC-III), as well as lower rates of nondaily smokers (NSDUH).

\subsection{Propensity Scores}

Propensity scores modeling the probability of being in ACCENT, based upon the baseline characteristics, are shown in Table 3. The propensity scores are used to measure the differences between the baseline characteristics of the ACCENT sample and the national sample. $\Delta$ p ranged from 0.0007 for NESARC-III to 0.0343 for TEDS-A, and standardized $\Delta \mathrm{p}$ was 1.72 standard deviations for TEDS-A, 3.65 standard deviations for NSDUH, and 6.67 standard deviations for NESARC-III. These values exceed the 0.25 standardized $\Delta \mathrm{p}$ cut-off, which indicates significant differences between ACCENT and the national samples with respect to the demographics and cannabis use characteristics considered.

\section{Discussion}

Demographic, cannabis, and tobacco use characteristics were found to be different among a cannabis cessation pharmacotherapy clinical trial sample conducted in the US compared to national datasets of current adult cannabis users with CUD. The clinical trial sample from ACCENT tended to be older; had more representation of African American and Hispanic/ Latino ethnicities; was more educated; and was more likely to be married (or in a domestic partnership). The ACCENT sample had a greater representation of daily or almost daily cannabis use, and a lower proportion of tobacco use.

Propensity score calculations showed that standardized $\Delta \mathrm{p}$ values were far beyond the proposed cut-off for sample similarity (0.25) (Cochran and Rubin, 1973; Rosenbaum and Rubin, 1985), demonstrating that the ACCENT sample was significantly different from national datasets. The TEDS-A dataset yielded the lowest standardized $\Delta \mathrm{p}$, indicating this population was the most similar to the ACCENT sample compared to the household surveys, but still well beyond the cut-off. Differences may be expected between the TEDS-A and the NSDUH/NESARC-III, given that the latter are household surveys and most cannabis users interviewed for those surveys were not necessarily seeking treatment for their drug use. As such, these national datasets represent unique populations of cannabis users, but none demonstrated similarity to the ACCENT study sample.

These results are consistent with the Susukida and colleagues paper (2016), which found differences within diverse CTN study samples and nationally representative samples, specifically in the areas of education and employment. Similar to Susukida and colleagues (2016), the current study found higher levels of education within the ACCENT sample compared to national samples, though employment seemed to fall in between the TEDS-A dataset (greater employment and less unemployment) and household surveys (less employed and greater unemployment). 
An encouraging finding from this analysis was that the ACCENT study recruited a more diverse racial and ethnic study sample. Racial and ethnic diversity of the sample was a study priority; sites were selected and recruitment strategies were modified as needed throughout enrollment in order to promote such diversity. Though males made up the majority of study participants in ACCENT, this accurately reflects national estimates of cannabis users, with nearly two-thirds of those with CUD or seeking treatment for cannabis use being male.

The differences found from this analysis are not surprising given that sample representativeness of clinical trials has been questioned (Humphreys, 2016; Rothwell, 2005), but does illustrate the need to ensure representative samples for CUD clinical trials. There are several potential avenues to improve sample representation. Alternative methods of recruitment could be used to target populations that may not be as likely to engage in clinical research, including those who are court-mandated to treatment for cannabis use as they represent a substantial portion of those enrolled in treatment programs. Proactive methods of recruitment could be used to contact a diverse population that might otherwise not contact study teams through advertisements. For large clinical trials, multiple study sites are often used and care should be taken in selecting these sites based on the patient/participant population. The National Drug Abuse Treatment CTN is a network of affiliated research centers, clinics, and collaborators, all with demographic data on the population of their clinics or their previous recruitment performance. This is an advantage to conducting clinical trials within the CTN platform, but all multi-site studies should strive for appropriate sites and adequate diversity of the study sample. The cannabis using population and those meeting for cannabis use disorder are a heterogeneous group and study samples should adequately reflect that.

Stringent inclusion/exclusion criteria continue to be a barrier to sample representativeness. Exclusion criteria employed by most clinical trials impacts the generalizability and representativeness of the sample, which has been demonstrated among those with cannabis dependence in a community sample (Okuda et al., 2010), for other substances of abuse (Blanco et al., 2008; Le Strat et al., 2011; Motschman et al., 2016), and among other medical disorders (Humphreys et al., 2013). Studies should consider more inclusive criteria to improve sample representativeness, when appropriate based on the phase of the study and other potential factors (e.g., risks of study intervention). None of the proposed solutions to improve sample representativeness are without challenges and inherent disadvantages, but this should be an important consideration when designing CUD treatment trials moving forward.

\subsection{Limitations}

This study has several limitations. First, the timeframe of data collection for the ACCENT study and for all national datasets does not perfectly align to capture the same months or years. However, all data used for comparisons were the most recent available datasets at the time of this analysis. Second, the TEDS-A data only captures admissions to SUD treatment clinics that received federal funds, thus excluding data from private facilities and a potentially important group of cannabis users. Additionally, TEDS-A data includes both those who were court-mandated to treatment compared to entering treatment on a more 
voluntary basis. Third, comparisons were made between national datasets and the ACCENT study sample on demographic and clinical characteristics that were captured in a uniform manner across surveys and could be categorized to be comparable. As such, a limited number of characteristics were compared in this analysis and used in determining propensity scores. Only observed characteristics can be accounted for using this statistical method.

\subsection{Conclusions}

The results from this analysis contribute to the body of literature suggesting that those who participate in research and specifically randomized clinical trials may be different, in terms of demographics and clinical characteristics, compared to national samples of the target population. The sample from the ACCENT trial appear to be unique from both SUD treatment admission data and household surveys, the latter of which may be more likely to capture users of lower severity, higher functioning, and non-treatment seeking/accepting. This may explain why the TEDS-A dataset and the ACCENT sample were closer in propensity scores than the two community samples. Notably, this study was specific to heavy cannabis users interested in quitting, which may be a population that will increase over the next few years given changes in public perception and the regulatory status of cannabis. Considering sample representativeness must be a key element through protocol development, choice of exclusion criteria, and throughout trial enrollment in order to best represent the clinical population in treatment studies and improve the generalizability of promising treatment strategies.

\section{References}

Berg CJ, Stratton E, Schauer GL, Lewis M, Wang Y, Windle M, Kegler M. Perceived harm, addictiveness, and social acceptability of tobacco products and marijuana among young adults: marijuana, hookah, and electronic cigarettes win. Subst. Use Misuse. 2015; 50:79-89. [PubMed: 25268294]

Blanco C, Olfson M, Okuda M, Nunes EV, Liu SM, Hasin DS. Generalizability of clinical trials for alcohol dependence to community samples. Drug Alcohol Depend. 2008; 98:123-128. [PubMed: 18579319]

Brady JE, Li G. Trends in alcohol and other drugs detected in fatally injured drivers in the United States, 1999-2010. Am. J. Epidemiol. 2014; 179:692-699. [PubMed: 24477748]

Center for Behavioral Health Statistics and Quality. Behavioral health trends in the United States: Results from the 2014 National Survey on Drug Use and Health (HHS Publication No. SMA 15-4927, NSDUH Series H-50). 2015. http://www.samhsa.gov/data.accessed on June 23, 2016

Cochran WG, Rubin DB. Controlling Bias in Observational Studies: A Review. The Indian Journal of Statistics, Series A (1961-2002). 1973; 35:417-446.

Compton WM, Gfroerer J, Conway KP, Finger MS. Unemployment and substance outcomes in the United States 2002-2010. Drug Alcohol Depend. 2014; 142:350-353. [PubMed: 25042761]

First, MSR., Gibbon, M., Williams, JBW. Biometrics Research. New York State Psychiatric Institute; New York: 1994. Structured Clinical Interview for DSM-IV-TR Axis I Disorders, Research Version, Patient Edition. (SCID-I/P).

Grant, BF., Chu, A., Sigman, R., Amsbary, M., Kali, J., Sugawara, Y., Jiao, R., Ren, W., Goldstein, R. Source and Accuracy Statement: National Epidemiologic Survey on Alcohol and Related Conditions-III (NESARC-III). Rockville, MD: 2014.

Gray KM, Sonne SC, McClure EA, Ghitza UE, Matthews A, McRae-Clark AL, Carroll KM, Potter J, Wiest K, Mooney LJ, Hasson AL, Walsh SL, Lofwall MR, Babalonis S, Lindblad R, Sparenborg S, Wahle A, King JS, Baker NL, Tomko RL, Haynes L, Vandrey R, Levin FR. A Randomized Placebo- 
Controlled Trial of N-Acetylcysteine for Cannabis Use Disorder in Adults. Drug Alcohol Depend. Under Review.

Grucza RA, Agrawal A, Krauss MJ, Cavazos-Rehg PA, Bierut LJ. Recent Trends in the Prevalence of Marijuana Use and Associated Disorders in the United States. JAMA psychiatry. 2016; 73:300301. [PubMed: 26864618]

Hall W. The adverse health effects of cannabis use: what are they, and what are their implications for policy? The International journal on drug policy. 2009; 20:458-466. [PubMed: 19362460]

Hall W, Degenhardt L. Adverse health effects of non-medical cannabis use. Lancet. 2009; 374:13831391. [PubMed: 19837255]

Hall W, Lynskey M. Evaluating the public health impacts of legalizing recreational cannabis use in the USA. Addiction. 2016

Hasin DS, Kerridge BT, Saha TD, Huang B, Pickering R, Smith SM, Jung J, Zhang H, Grant BF. Prevalence and Correlates of DSM-5 Cannabis Use Disorder, 2012-2013: Findings from the National Epidemiologic Survey on Alcohol and Related Conditions-III. A. J. Psychiatry. 2016; 173:588-599.

Hasin DS, Saha TD, Kerridge BT, Goldstein RB, Chou SP, Zhang H, Jung J, Pickering RP, Ruan WJ, Smith SM, Huang B, Grant BF. Prevalence of Marijuana Use Disorders in the United States Between 2001-2002 and 2012-2013. JAMA psychiatry. 2015; 72:1235-1242. [PubMed: 26502112]

Humphreys K. Grappling with the generalizability crisis in addiction treatment research. Addiction. 2016; 111:1141-1142. [PubMed: 27079280]

Humphreys K, Maisel NC, Blodgett JC, Finney JW. Representativeness of patients enrolled in influential clinical trials: a comparison of substance dependence with other medical disorders. J. Stud. Alcohol Drugs. 2013; 74:889-893. [PubMed: 24172115]

Johnston, LD., O'Malley, PM., Meiech, RA., Bachman, JG., Schulenberg, JE. Monitoring the Future national results on drug use: 1975-2014: Overview, key findings on adolescent drug use. Institute for Social Research, University of Michigan; Ann Arbor, MI: 2015.

Le Strat Y, Rehm J, Le Foll B. How generalisable to community samples are clinical trial results for treatment of nicotine dependence: a comparison of common eligibility criteria with respondents of a large representative general population survey. Tob. Control. 2011; 20:338-343. [PubMed: 21212379]

Lynskey M, Hall W. The effects of adolescent cannabis use on educational attainment: a review. Addiction. 2000; 95:1621-1630. [PubMed: 11219366]

McClure EA, Sonne SC, Winhusen T, Carroll KM, Ghitza UE, McRae-Clark AL, Matthews AG, Sharma G, Van Veldhuisen P, Vandrey RG, Levin FR, Weiss RD, Lindblad R, Allen C, Mooney LJ, Haynes L, Brigham GS, Sparenborg S, Hasson AL, Gray KM. Achieving cannabis cessation -evaluating $\mathrm{N}$-acetylcysteine treatment (ACCENT): design and implementation of a multi-site, randomized controlled study in the National Institute on Drug Abuse Clinical Trials Network. Contemp. Clin. Trials. 2014; 39:211-223. [PubMed: 25179587]

Meier MH, Caspi A, Ambler A, Harrington H, Houts R, Keefe RS, McDonald K, Ward A, Poulton R, Moffitt TE. Persistent cannabis users show neuropsychological decline from childhood to midlife. Proc. Natl. Acad. Sci. U.S.A. 2012; 109:E2657-2664. [PubMed: 22927402]

Motschman CA, Gass JC, Wray JM, Germeroth LJ, Schlienz NJ, Munoz DA, Moore FE, Rhodes JD, Hawk LW, Tiffany ST. Selection criteria limit generalizability of smoking pharmacotherapy studies differentially across clinical trials and laboratory studies: A systematic review on varenicline. Drug Alcohol Depend. 2016; 169:180-189. [PubMed: 27863344]

Okuda M, Hasin DS, Olfson M, Khan SS, Nunes EV, Montoya I, Liu SM, Grant BF, Blanco C. Generalizability of clinical trials for cannabis dependence to community samples. Drug Alcohol Depend. 2010; 111:177-181. [PubMed: 20537813]

Pacek LR, Mauro PM, Martins SS. Perceived risk of regular cannabis use in the United States from 2002 to 2012: differences by sex, age, and race/ethnicity. Drug Alcohol Depend. 2015; 149:232244. [PubMed: 25735467]

Rosenbaum PR, Rubin DB. Constructing a control group using multivariate matched sampling methods that incorporate the propensity score. The American Statistician. 1985; 39:33-38. 
Rothwell PM. External validity of randomised controlled trials: "to whom do the results of this trial apply?". Lancet. 2005; 365:82-93. [PubMed: 15639683]

Sinclair CF, Foushee HR, Scarinci I, Carroll WR. Perceptions of harm to health from cigarettes, blunts, and marijuana among young adult African American men. J. Health Care Poor Underserved. 2013; 24:1266-1275. [PubMed: 23974397]

Stuart EA. Matching methods for causal inference: A review and a look forward. Statistical science: a review journal of the Institute of Mathematical Statistics. 2010; 25:1-21. [PubMed: 20871802]

Stuart EA, Cole SR, Bradshaw CP, Leaf PJ. The use of propensity scores to assess the generalizability of results from randomized trials. Journal of the Royal Statistical Society. Series A, (Statistics in Society). 2001; 174:369-386.

Substance Abuse and Mental Health Services Administration; Center for Behavioral Health Statistics and Quality. Treatment Episode Data Set (TEDS): 2003-2013. In: Administration, S.A.a.M.H.S. , editor. National Admissions to Substance Abuse Treatment Services. Rockville, MD: 2015.

Susukida R, Crum RM, Stuart EA, Ebnesajjad C, Mojtabai R. Assessing Sample Representativeness in Randomized Control Trials: Application to the National Institute of Drug Abuse Clinical Trials Network. Addiction. 2016

United States Department of Health and Human Services Substance Abuse and Mental Health Services Administration. National Survey on Drug Use and Health, 2014. U.S. Department of Health and Human Services, Substance Abuse and Mental Health Services Administration; Ann Arbor, MI: 2016.

Volkow ND, Swanson JM, Evins AE, DeLisi LE, Meier MH, Gonzalez R, Bloomfield MA, Curran HV, Baler R. Effects of Cannabis Use on Human Behavior, Including Cognition, Motivation, and Psychosis: A Review. JAMA psychiatry. 2016; 73:292-297. [PubMed: 26842658] 
Table 1

Weighted and unweighted sample sizes for CTN-0053 (ACCENT), NSDUH, NESARC-III, and TEDS-A.

\begin{tabular}{|l|c|c|c|c|}
\hline & ACCENT & NSDUH & NESARC-III & TEDS-A \\
\hline Raw N & & $265,122,864$ & $235,411,957$ & \\
\hline Raw N (unweighted) & 302 & 55,271 & 36,309 & $1,683,451$ \\
\hline Sample Subset N & & $1,832,081$ & $4,765,117$ & \\
\hline Sample Subset N (unweighted) & 296 & 524 & 766 & 28,955 \\
\hline
\end{tabular}

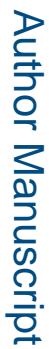

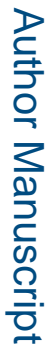

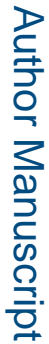




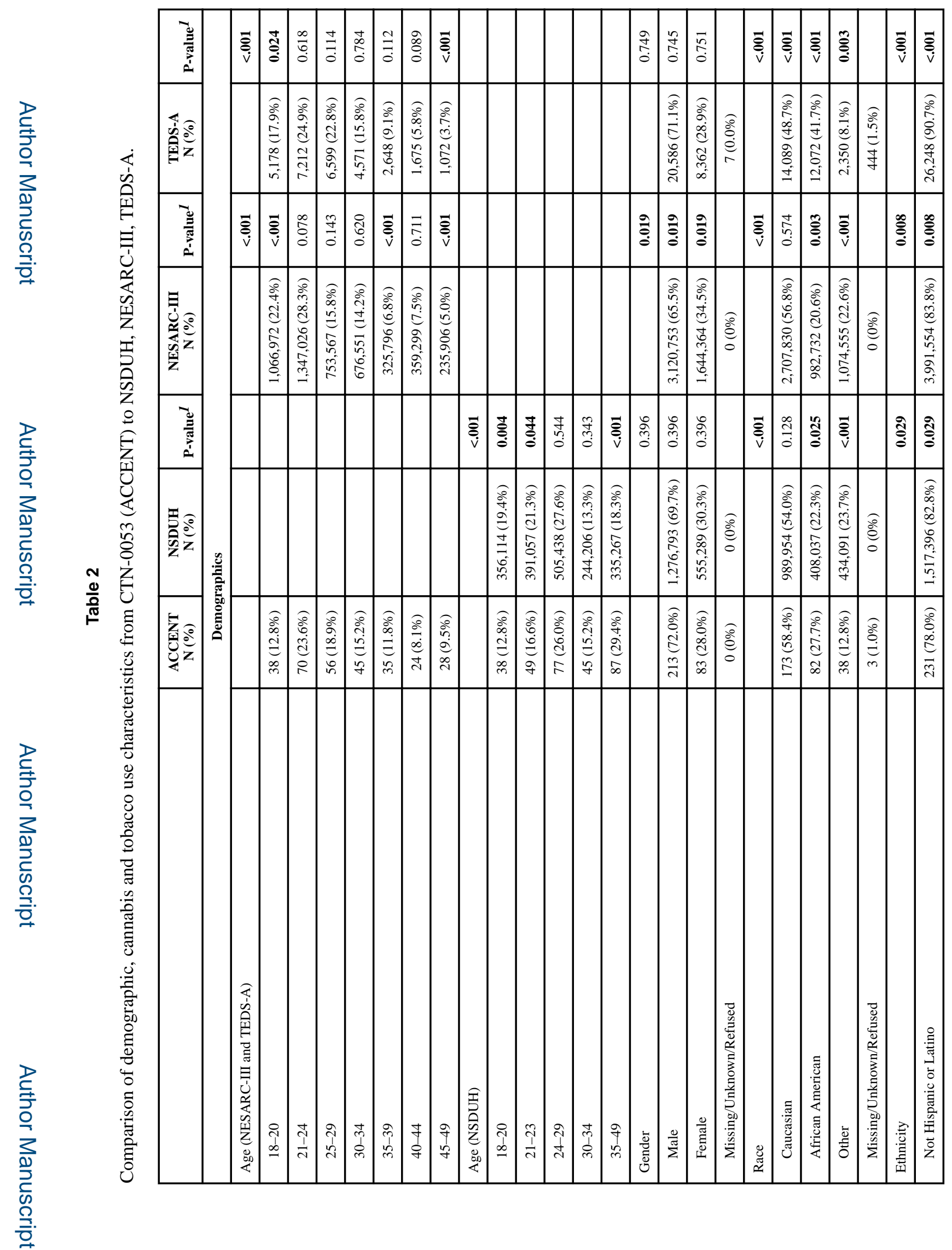

Drug Alcohol Depend. Author manuscript; available in PMC 2017 September 06. 


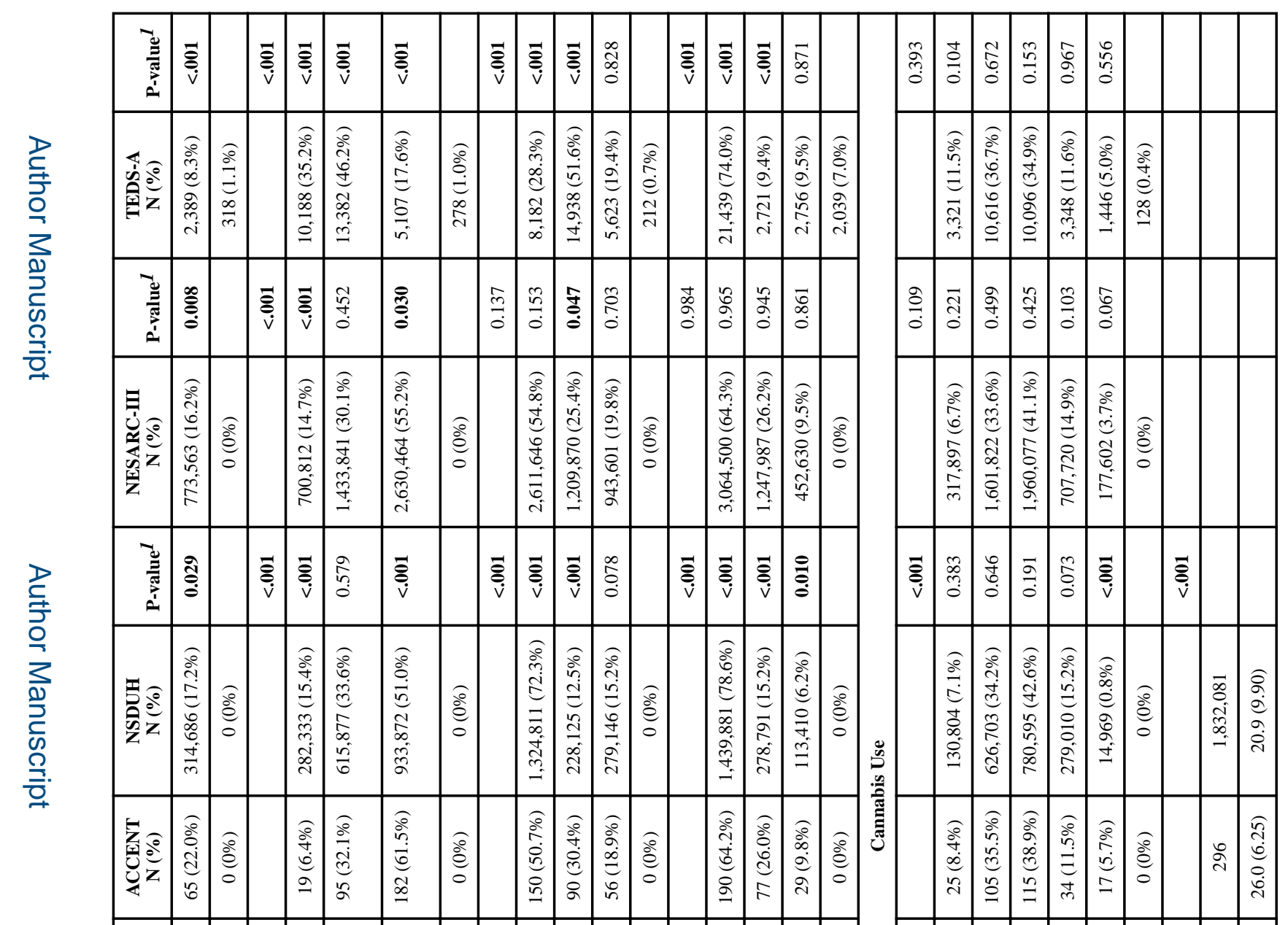

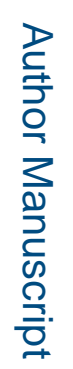

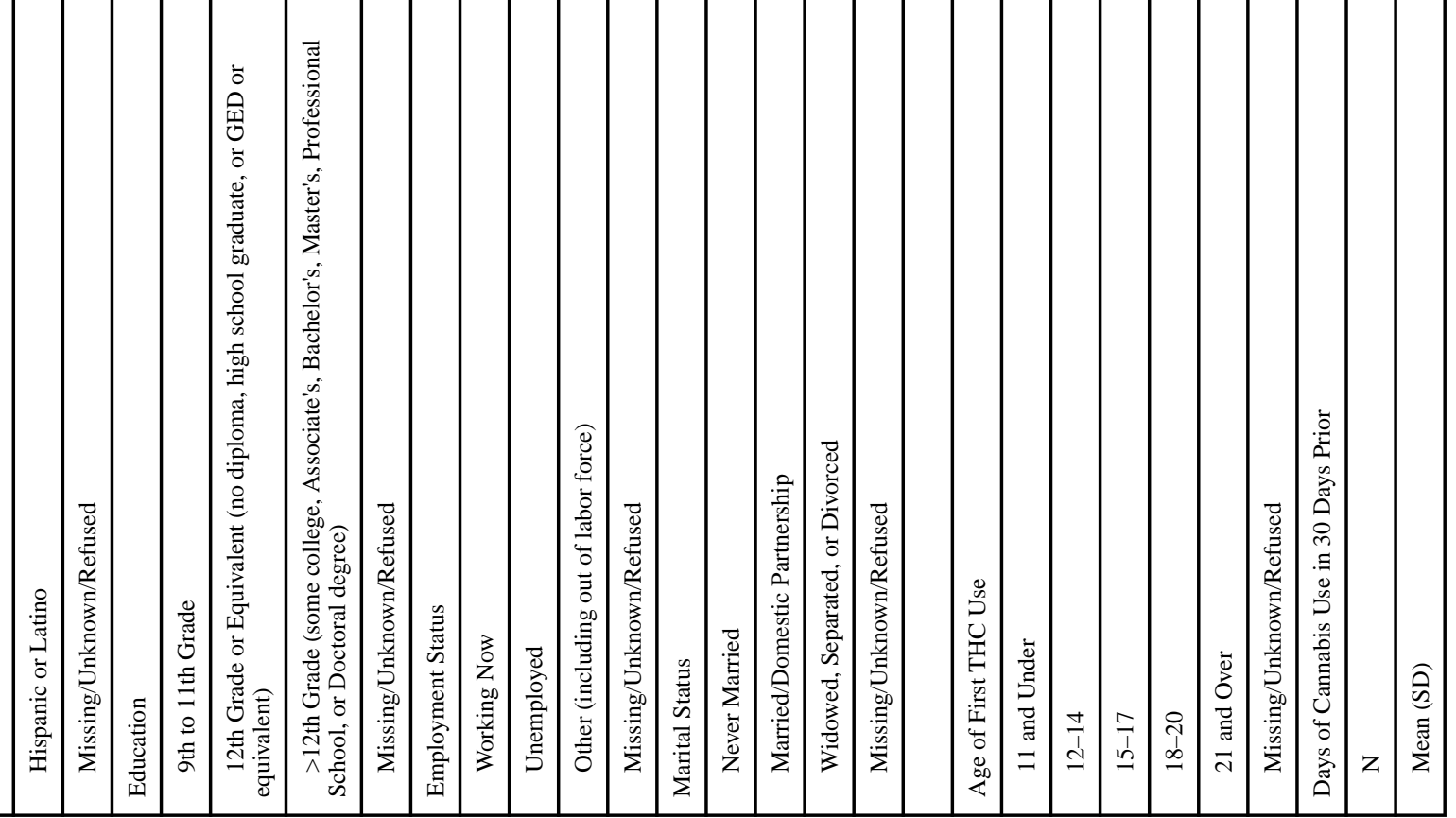

Drug Alcohol Depend. Author manuscript; available in PMC 2017 September 06. 


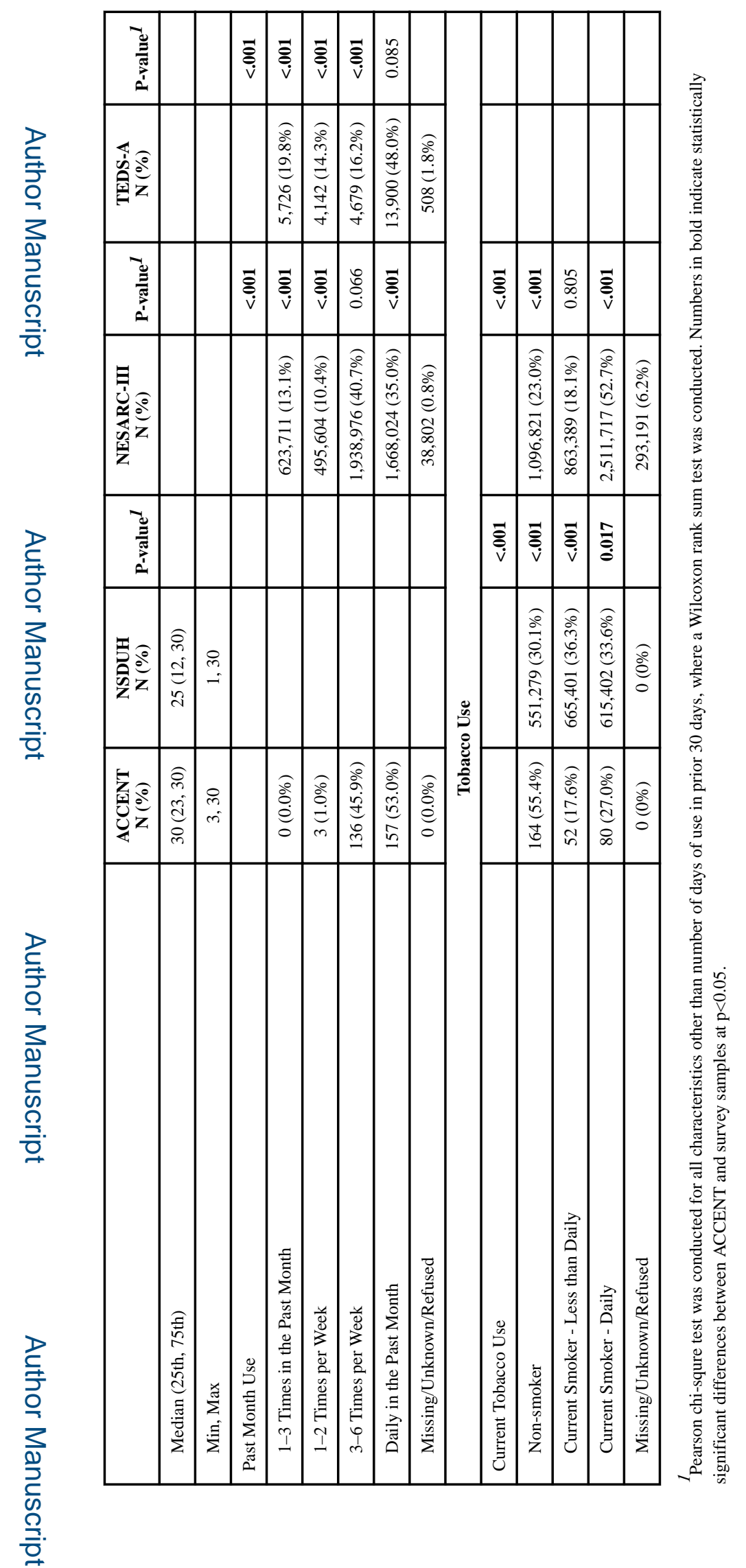

Drug Alcohol Depend. Author manuscript; available in PMC 2017 September 06. 


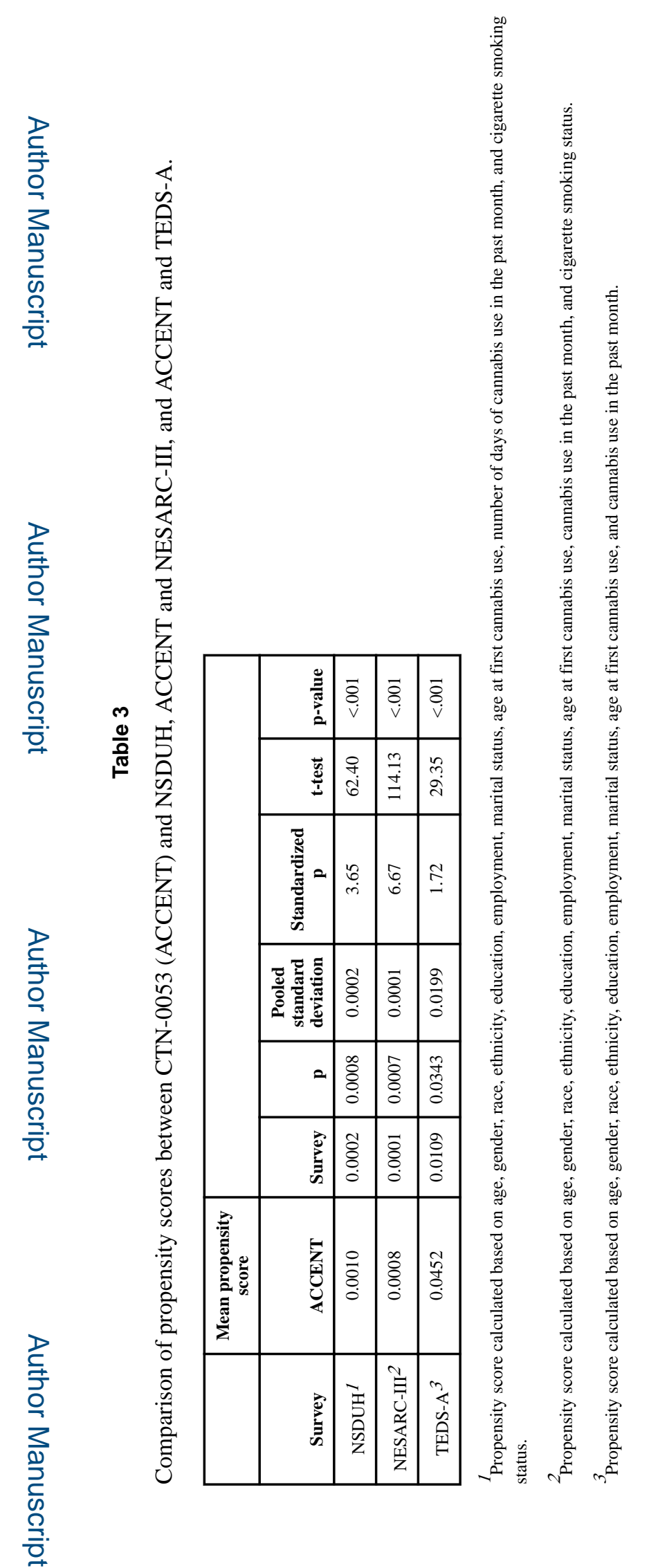

Drug Alcohol Depend. Author manuscript; available in PMC 2017 September 06. 